\title{
Eine Methode zum direkten Nachweis von Ammoniak im Seewasser
}

\author{
Von Max Gillbricht \\ Aus der Biologischen Anstalt Helgoland \\ (Mit 4 Abbildungen im Text)
}

Die früher angewandten Verfahren zur direkten Bestimmung des Ammoniaks im Seewasser mit dem Nessler-Reagens (NüManN, 1949) erwiesen sich als unbefriedigend. So wurden in den letzten Jahren drei neue Methoden vorgeschlagen:

1. BULJAN (1951): $\mathrm{NH}_{3}$ wird in alkalischer Lösung mit Hypobromit oxydativ zerstört, und anschließend das überschüssige $\mathrm{OBr}^{\prime}$ durch Oxydation von Bordeauxrot in saurer Lösung bestimmt.

2. Riley und Sinhaseni (1957): $\mathrm{NH}_{3}$ wird vom Seewasser durch Diffusion abgetrennt und dann mit Phenol zu Phenolblau umgesetzt (Hypochlorit als Oxydationsmittel). Die Methode hat die Nachteile, daß die Ausbeute bei der Diffusion schwankt, und daß die Stärke der Farbreaktion sehr von den Außenbedingungen abhängt, also ständig überprüft werden muß.

3. Strickland und Parsons (1960): Seewasser wird bei pH 3,6 in Gegenwart von Pyridin mit Chloramin T und einem Pyrazolon-Reagens versetzt; anschließend wird der gebildete Farbstoff mit $\mathrm{CCl}_{4}$ ausgeschüttelt, diese Lösung mit Hilfe eines Papierfilters gereinigt und dann photometriert. Das Verfahren ist umständlich und störanfällig.

Die Methode 1 dürfte demnach die weitaus einfachste sein. Dafür hat sie jedoch vor allem den Nachteil, daß in saurer Lösung nicht nur das Bordeauxrot, sondern zahlreiche oxydierbare Stoffe angegriffen werden. Dies sind im Seewasser in erster Linie die gelösten organischen Substanzen. Diese Störung wird schon bei der Arbeitsvorschrift von BULJAN (1951) weitgehend beseitigt, jedoch erwiesen sich die weiteren Angaben nicht als geeignet, brauchbare Messungen zu erzielen. Für unsere eigene Arbeit waren vielmehr vor allem die Untersuchungen von KOROLEFF (1959) nützlich.

Nach unseren Beobachtungen ist es gut, die Seewasser- und Reagenzienmengen nicht zu klein zu wählen. So lassen sich die Reagenzien relativ genau pipettieren (Knudsenpipette), und hiervon hängt weitgehend die Genauigkeit der Methode überhaupt ab.

$\mathrm{Zu}$ jeder Bestimmung gehören

a) Die Messung: Eine alkalische NaOBr-Lösung wird der Probe zugesetzt und zerstört so das Ammoniak. Das überschüssige Hypobromit reagiert an- 
schließend in saurer Lösung mit Bordeauxrot. Es wird also weniger Farbstoff verbraucht, wenn mehr $\mathrm{NH}_{3}$ vorhanden ist.

b) Die umgekehrte Zugabe: Es wird zuerst die saure Bordeauxrotlösung zugesetzt und dann erst das Hypobromit. So wirkt die gesamte Hypobromitmenge zerstörend auf das Bordeauxrot.

Lösung $b$ ist mithin schwächer gefärbt als Lösung $a$. Dieser Unterschied ist ein unmittelbares Maß für die Ammoniakmenge. Dieses Verfahren ist recht umständlich, aber nur so lassen sich Störungen durch andere oxydierbare Stoffe befriedigend vermeiden. Glaubt man unter besonderen Voraussetzungen, auf eine umgekehrte Zugabe für jede einzelne Probe verzichten zu können, so ist dann zu beachten, daß die Hypobromitlösung sich ständig zersetzt (vor allem in den ersten Stunden nach dem Ansetzen). Ihre Stärke muß also häufig überprüft werden. Soll eine Probe vor der Aufarbeitung einige Zeit gelagert werden, so geschieht dies nach unseren Beobachtungen zweckmäßig in Glasflaschen (nach Strickland und Parsons [1960] eignen sich auch Gefäße aus Polyäthylen) in der Tiefkühltruhe $\left(-16^{0}\right)$. Sofortige Verarbeitung oder wenigstens möglichst kurzzeitige Lagerung ist jedoch anzustreben, obschon bei unseren Versuchen innerhalb von zwei Wochen keine Veränderungen zu beobachten waren. Da diese Bestimmungen mit mehrfach neu angesetzten $\mathrm{NaOBr}-\mathrm{Lösun}-$ gen durchgeführt werden mußten, zeigen sie gleichzeitig, daß diese Tatsache die Meßergebnisse nicht beeinflußt.

Unter Berücksichtigung aller dieser Uberlegungen und Beobachtungen sieht die Arbeitsvorschrift wie folgt aus :

$$
\text { Reagenzien }
$$

Alle Lösungen sind in braunen Flaschen bzw. im Dunkeln aufzubewahren.

1) ca. $0,1 \mathrm{n} \mathrm{NaOBr}-$ Stammlösung

Herstellung: $1,25 \mathrm{ml}$ flüssiges Brom und $2,5 \mathrm{~g} \mathrm{NaOH}$ werden mit $\mathrm{H}_{2} \mathrm{O}$ auf $500 \mathrm{ml}$ aufgefüllt. Die Lösung ist haltbar.

2) ca. 0,002 n NaOBr-Gebrauchslösung

Herstellung: $2 \mathrm{ml}$ Stammlösung werden mit $0,3 \mathrm{n} \mathrm{NaOH}$ auf $100 \mathrm{ml}$ aufgefüllt. Die Lösung ist instabil und ändert sich in den ersten Stunden besonders stark. Sie sollte daher möglichst täglich frisch angesetzt werden und hält sich höchstens zwei Tage.

3) Bordeauxrotlösung

Herstellung: $200 \mathrm{mg}$ Bordeauxrot werden in $250 \mathrm{ml} \mathrm{0,6} \mathrm{n} \mathrm{CH}_{3} \mathrm{COOH}$ gelöst. Die Lösung ist mehrere Wochen haltbar.

\section{Messung}

1) Zweimal $50 \mathrm{ml}$ Seewasser werden unmittelbar nach der Gewinnung in saubere, trockene Glasstopfenflaschen pipettiert und dann sof ort weiterverarbeitet. Sollte dies nicht möglich sein, so läßt sich die Probe durch Aufbewahrung in der Tiefkühltruhe wenigstens für zwei Wochen (wahrscheinlich für erheblich länger) fixieren. 
2a) Es werden $2 \mathrm{ml} 0,002 \mathrm{n} \mathrm{NaOBr}$ zugesetzt, dann wird die Probe umgeschwenkt.

3a) 10 Minuten später werden $2 \mathrm{ml}$ Bordeauxrotlösung zugegeben. Anschließend wird umgeschwenkt, und die Probe bleibt für 30 (20 bis 60) Minuten im Dunkeln stehen.

\section{$\mathrm{S} \%$}

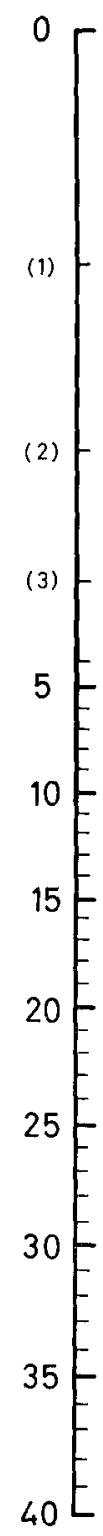

2b) Die Probe wird mit $2 \mathrm{ml}$ Bordeauxrotlösung versetzt, umgeschwenkt und sofort weiter verarbeitet, indem

3b) $2 \mathrm{ml} \mathrm{0,002} \mathrm{n} \mathrm{NaOBr}$ zugegeben werden. Anschließend wird umgeschwenkt, und die Probe bleibt für 30 (20 bis 60) Minuten im Dunkeln stehen.
$K \%$

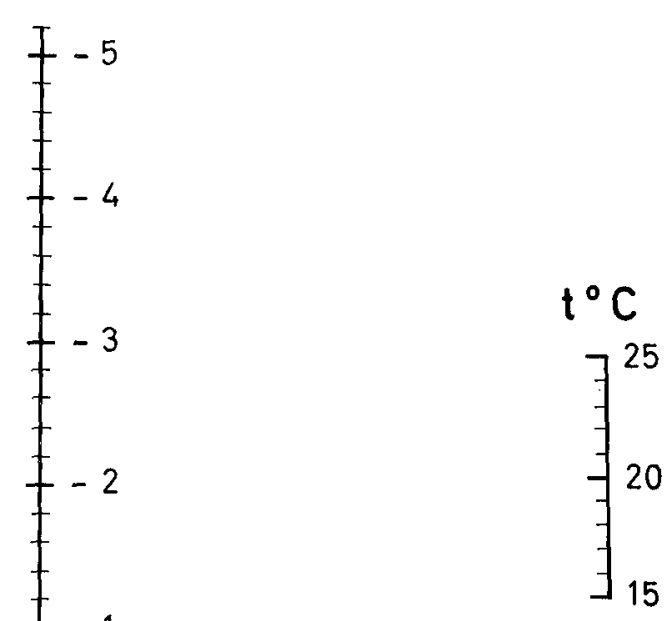

Abb. 1: Fluchtentafel zur Korrektur der unmittelbar erhaltenen Extinktionswerte (siehe Text) 
4) Nunmehr wird der Extinktionsunterschied $(\triangle \mathrm{EK})$ zwischen Probe a und Probe $b$ bei ca. $525 \mathrm{~m} \mu$ bestimmt. Zweckmäßig werden beide Proben direkt gegeneinander gemessen. Wir benutzen hierzu wegen der großen Empfindlichkeit und guten Reproduzierbarkeit der Meßbedingungen ein Filterphotometer (Elko II, Zeiss) und messen in I-cm-Küvetten unter Vorschaltung des Filters $\mathrm{S} 53 \mathrm{E}$.

Der so erhaltene Wert ist je nach Temperatur und Salzgehalt zu korrigieren. Wählt man $t=20^{\circ}$ und $S=35 \%$ als Bezugsgrößen, so ergeben sich aus der beigegebenen Fluchtentafel (Abb. 1) durch gerade Verbindung von $\mathbf{t}^{0}$ und $\mathrm{S} \% 0$ (Lineal) die zugehörigen Korrekturwerte in $\%$.

$$
\text { Beispiel: } \begin{aligned}
\triangle \mathrm{EK} & =0,0476 \\
\mathrm{t}^{0} & =24,5 \\
\mathrm{~S} \% & =16,7 \\
\mathrm{~K} \% & =-1,9
\end{aligned}
$$$$
\Delta \mathrm{EK}_{\mathrm{kor},}=0,0467
$$

Die Korrekturen für die eingeklammerten $S \% 0$-Werte sind unsicher, da in diesem Bereich keine Beobachtungen vorliegen und daher bei Festlegung dieser Daten von fragwürdigen Annahmen ausgegangen werden muß.

Innerhalb des Meßbereichs ist $\triangle \mathrm{EK}$ eine lineare Funktion des Ammoniakgehalts, und unter unseren Arbeitsbedingungen besteht folgender Zusammenhang: $\quad 1 \mu \mathrm{g} \mathrm{NH}{ }^{-}-\mathrm{N} / 1=0,00076 \triangle \mathrm{EK}$

Der Meßbereich geht von 0 bis $200 \mu \mathrm{g} \mathrm{NH}_{4}{ }_{4}-\mathrm{N} / \mathrm{l}$, bei frischen $\mathrm{NaOBr}-\mathrm{Lö}-$ sungen mögen auch Mengen bis $250 \mu \mathrm{g}$ noch zuverlässig nachweisbar sein.

Die mittlere Streuung dürfte zumeist kleiner als $\pm 4 \mu \mathrm{g} \mathrm{NH}{ }^{-}{ }_{4} \mathrm{~N} / 1$ sein, kann jedoch bei großen Ammoniakmengen bis $\pm 5 \mu \mathrm{g} \mathrm{NH}{ }^{-}-\mathrm{N} / 1$ ansteigen. Die aus der Fluchtentafel zu entnehmenden Korrekturwerte übersteigen also nur selten die methodische Streuung. Die Korrekturen haben daher eine größere praktische Bedeutung nur dann, wenn die Salzgehalte sehr niedrig sind, oder wenn durch Mittelbildung auch kleinere systematische Fehler erkennbar werden.

Es ist nicht möglich, eine Ammoniakbestimmung störungsfrei in Gegenwart anderer (vor allem organischer $\mathrm{N}$-haltiger) Stoffe durchzuführen, ohne das $\mathrm{NH}_{3}$ zuvor durch Destillation oder Diffusion abzutrennen. Wird hierauf zur Vereinfachung verzichtet, dann muß mit Meßfehlern gerechnet werden. Störungen im sauren Bereich werden zwar durch die umgekehrte Zugabe weitgehend ausgeschaltet, aber eine Untersuchung über den Einfluß verschiedener Stoffe auf den $\mathrm{NH}_{3}$-Nachweis erschien lohnend und ist auch schon von BuLJAN (1951) durchgeführt worden. Wir erhielten bei unseren Messungen folgende Ergebnisse:

Stoff zugesetzte Menge Befund

\begin{tabular}{lrl}
\hline Nitrit & $28 \mu \mathrm{gN} / 1$ & kein Einfluß \\
Glucose & $4000 \mu \mathrm{g} / 1$ & kein Einfluß \\
Athanol & $20000 \mu \mathrm{g} / 1$ & kein Einfluß \\
Albumin & $100 \mu \mathrm{gN} / 1$ & $\begin{array}{l}\text { kein Einfluß, eine geringe Zunahme der } \\
\text { Extinktion dürfte auf Verunreinigung der }\end{array}$ \\
& & $\begin{array}{l}\text { Substanz zurückzuführen sein } \\
\text { Diäthylamin }\end{array}$ \\
Triäthanolamin & $140 \mu \mathrm{gN} / 1$ & kein Einfluß \\
$25 \%$ der N-Menge werden nachgewiesen
\end{tabular}


Stoff zugesetzte Menge Befund

Glykokoll $280 \mu \mathrm{gN} / 1 \quad 100 \%$ der $\mathrm{N}$-Menge werden nachgewiesen

Histidin $140 \mu \mathrm{gN} / \mathrm{l} \quad 100 \%$ des Gesamt-N werden nachgewiesen Tyrosin $140 \mu \mathrm{gN} / 1 \quad 150 \%$ der $\mathrm{N}$-Menge werden nachgewiesen

Die störende Wirkung einer organischen Substanz in \% ihres N-Gehaltes anzugeben, ist rein formal, da wir zunächst nicht wissen, wie das Hypobromit den Stoff angreift. Es sind daher sehr wohl Werte über 100\% möglich (Tyro$\sin )$.
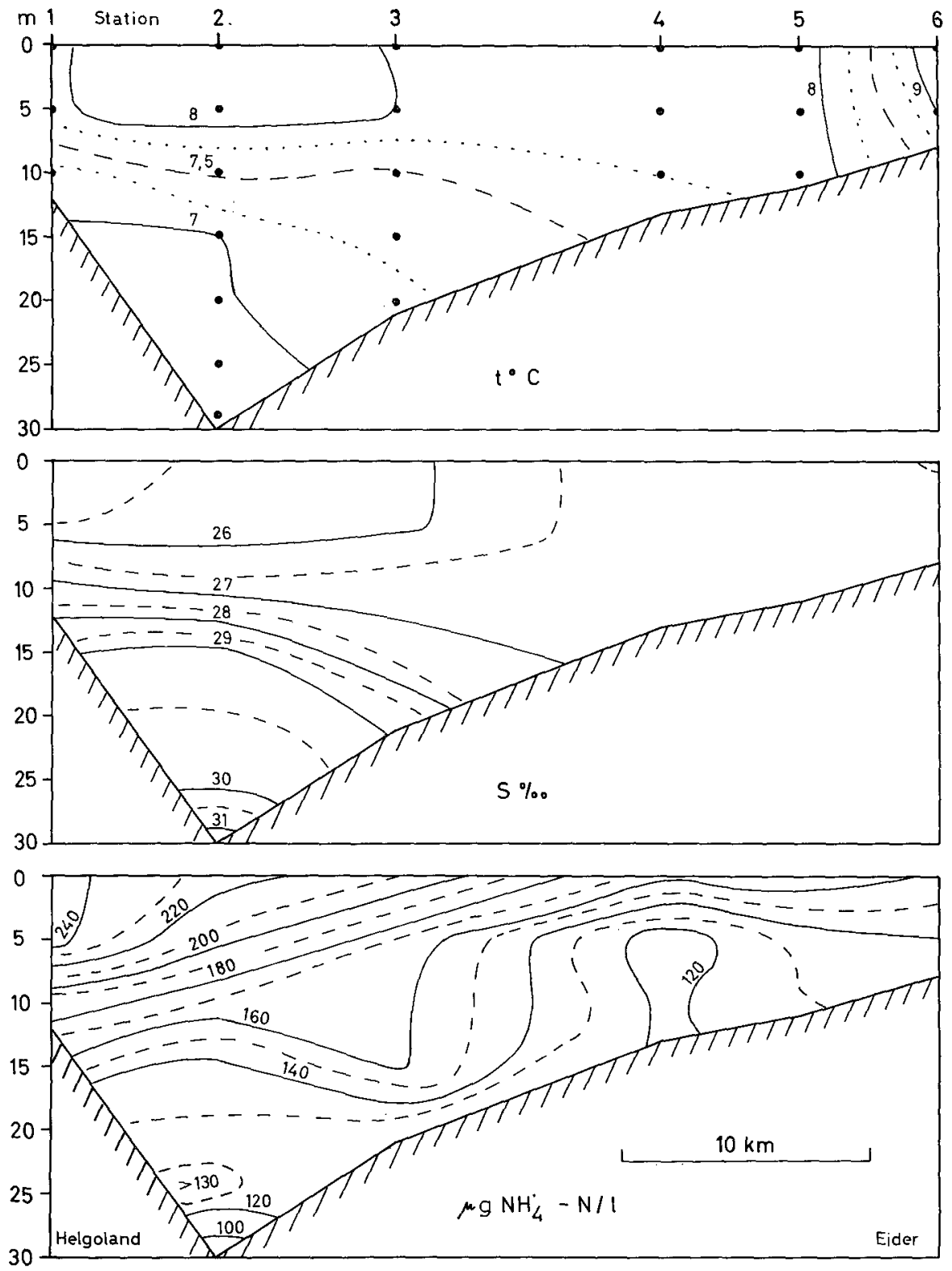

Abb. 2: Isoplethendiagramm der Verteilung von Temperatur $\left(t^{0}\right)$, Salzgehalt (S yos) und Ammoniakgehalt $\left(\mu \mathrm{g} \mathrm{NH}_{4}{ }^{-}-\mathrm{N} / 1\right)$ zwischen Helgoland und der Eidermündung am 17. 4. 1961 
Falls bei den im Seewasser gelösten organischen Substanzen den Aminosäuren eine größere Bedeutung zukommt, sind die mit diesem Verfahren erhaltenen "Ammoniakwerte" mit Vorsicht zu betrachten.

\section{Anwendungsbeispiel}

Am 17. April 1961 führte der F. K. „Uthörn" einen hydrographischen Schnitt von Helgoland zur Eidermündung durch (Abb. 2). Wie die Isoplethendiagramme zeigen, hatten wir es mit einer abnormen Lage zu tun. Schwache NO-Winde hatten innerhalb weniger Tage das Oberflächenwasser nach Westen gedrückt und gleichzeitig an der Küste ein Auftriebsgebiet geschaffen. Dies zeigen Temperatur, Salzgehalt und wohl am besten die Ammoniakmessungen. Demnach ist das Ammoniak vorzugsweise in der salzarmen Oberschicht zu Hause, und der Wind hat es von der Küste nach Helgoland getrieben.

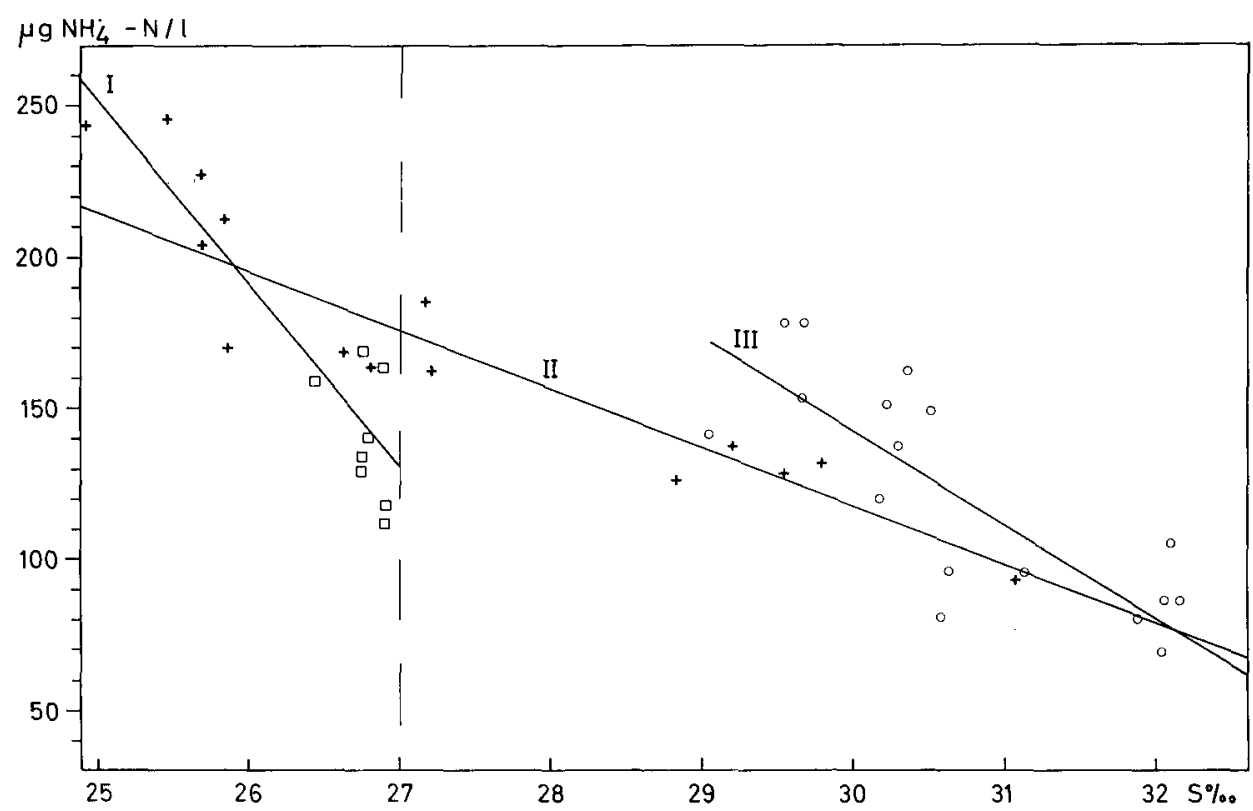

Abb. 3: Zusammenhang zwischen Ammoniak- und Salzgehalt

Gerade I : Küstenwasser am 17.4.1961 (S <27\%0)

Gerade II : Bodenwasser Station 1 bis 3 am 17. 4. 1961 (S $>27 \%$ )

Gerade III: Helgolandwasser am 13. 4. 1961

$+=$ Stat. 1 bis $3, \square=$ Stat. 4 bis $6, O=$ Proben vom 13. 4.1961

Es bleibt jetzt noch zu klären, wie eng die Bindung des Ammoniaks an die einzelnen Wasserkörper ist, oder ob es gar aus dem Süßwasser stammt. Um dies zu ermitteln, tragen wir zunächst die Ammoniakwerte gegen den Salzgehalt auf (Abb. 3). Wir erhalten so ein recht kompliziertes Bild: Die Tiefenwerte der Stationen 1 bis 3 liegen nicht auf einer Geraden mit denen der Oberfläche. Man hat vielmehr den Eindruck, daß sich am Boden ein Wasser befindet, das durch Mischen des untersten Oberflächenwassers mit einem noch unbekannten Wasser entstanden ist; es dürfte sich also um einen recht neuen Zustand handeln. Die lineare Beziehung des Oberflächenwassers zu 
seinem früheren Tiefenwasser (jetzt auf den Stationen 4 bis 6 die gesamte Wassersäule ausfüllend) ist aber noch vorhanden. Nun liegt es nahe, als unbekannten Wasserkörper das vorher bei Helgoland vorhanden gewesene Wasser anzunehmen, das noch am 13. 4. 1961 rings um die Insel nachgewiesen wurde. Die Eintragung dieser Werte in das Diagramm dürfte diese Vermutung bestätigen. Die Gerade II zeigt also die Vermischung zweier bisher voneinander unabhängiger Wasserkörper (I und III) an.

Das t/S-Diagramm ergibt ein ähnliches Bild, jedoch hat sich das frühere Tiefenwasser auf den Stationen 4 bis 6 zu stark erwärmt, so daß es sich der Geraden I nicht mehr einfügt. Der Ammoniakgehalt hat sich also bei dieser Beobachtung als konservativer erwiesen als die Temperatur.

Woher kommen aber diese Ammoniakmengen? Nach Abb. 3 müssen wir sie als Funktion des Salzgehaltes auffassen, indem sich salzarme und ammoniakreiche Wassermassen mit salzreichen und ammoniakarmen vermischen. Theoretisch ist außerdem zu fordern, daß wegen der Oxydation des Ammoniaks dieses stärker ab- als der Salzgehalt zunimmt.

Tragen wir die Geraden I und III in ein neues Diagramm ein (Abb. 4a), so sollten wir durch Extrapolation wenigstens größenordnungsmäßig erkennen können, wie hoch (mindestens) der Ammoniakgehalt des zugehörigen Süßwassers gewesen sein muß, und mit welchem Seewasser (unter der Annahme der Ammoniakmenge 0 ) sich dieses vermischt hat. $\mathrm{Ob}$ diese Werte vertretbar sind, soll die Gerade IV zeigen, die sich aus den zur gleichen Jahreszeit 1957 bei Cuxhaven von BUrsche, KüHL und MANN (1958) ermittelten Daten ableiten läßt. Ihr Verlauf deutet darauf hin, daß sich das Wasser der Elbe zur Beobachtungszeit nicht direkt mit dem Nordseewasser, sondern zunächst mit dem des Wattengebietes vermischt hat, wie dies z. B. auch aus dem Gebiet der Jade bekannt ist (Grulbricht, 1956). Die Gerade III scheint ein Mischwasser zu repräsentieren, dessen einer Grenzwert reines Nordseewasser ist, während der andere irgendwie vom Elbwasser beeinflußt sein dürfte. Die Gerade I für das Küstenwasser ist von anderen Zuflüssen abhängig; dieses scheint sich auch nicht direkt mit dem Nordseewasser zu vermischen.

Aber geht die Oxydation des Ammoniaks nicht viel zu rasch, als daß man es noch bei Helgoland als mit dem Elbwasser verfrachtet bezeichnen könnte? Dann müßten sich diese Ammoniakmengen an Ort und Stelle gebildet haben; hierfür kämen am ehesten Nitratreduktion durch das Phytoplankton und um diese Jahreszeit wohl nur untergeordnet die Zersetzung von organischer Substanz in Frage, womit in erster Linie in der durchleuchteten Oberschicht zu rechnen wäre. Die Werte würden dann auch nach Eliminierung des Salzgehaltseinflusses mit der Tiefe abnehmen, während nach Eliminierung der Tiefe zum Salzgehalt nur eine unbedeutende Beziehung bestehen bleiben müßte.

Die vorliegenden Messungen liefern zu dieser Fragestellung folgende Ergebnisse:

\footnotetext{
rN.S.m $\quad=$ Anzahl der Messungen

$\mathrm{n} \quad=$ Korrelation zwischen Ammoniak- und Salzgehalt nach Eliminierung der Tiefe

$\mathrm{r}_{\mathrm{N}, \mathrm{m}, \mathrm{S}} \quad=$ Korrelation zwischen Ammoniakgehalt und Tiefe nach Eliminierung des Salzgehalts

$\mathrm{p}^{\%}=$ Unsicherheit in $\%$, daß eine Korrelation im angegebenen Sinne $(+$ oder $\rightarrow$ ) besteht

$\triangle \mathrm{N} / \mathrm{m}=$ Abnahme des $\mathrm{NH}_{4}{ }_{4} \mathrm{~N}[\mu \mathrm{g} / 1]$ mit der Tiefe [m] unter gesonderter Berücksichtigung des Salzgehalts (Mehrfachkorrelation)

$\Delta \sigma / 1000 \mathrm{~m}=$ Zunahme der Dichte mit der Tiefe
} 
Tabelle 1

n r.S.m $\quad \mathrm{p} \% \quad$ rN.m.S $\quad \mathrm{p}^{\%} / \mathrm{N} \quad \Delta \mathrm{N} / \mathrm{m} \quad \Delta \sigma_{\mathrm{t}} /$

$1000 \mathrm{~m}$

$\begin{array}{lllllllll}\text { 1) Helgolandwasser } & 13.4 . & 18 & -0,642 & <1 & -0,028 & \geqslant 5 & -0,07 & 55,9\end{array}$

$\begin{array}{lllllllll}\text { 2) Stat. } 1 \text { bis } 3 & \mathrm{~S}<27 \% & 8 & -0,663 & >5 & -0,182 & \$ 5 & -1,25 & 82,7\end{array}$

$\begin{array}{lllllllll}\text { 3) Stat. } 1 \text { bis } 3 & \mathrm{~S}>27 \% & 7 & -0,687 & >5 & -0,424 & >5 & -1,45 & 179,2\end{array}$

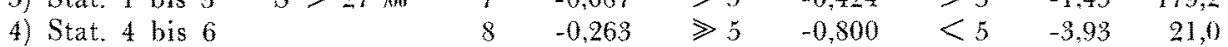

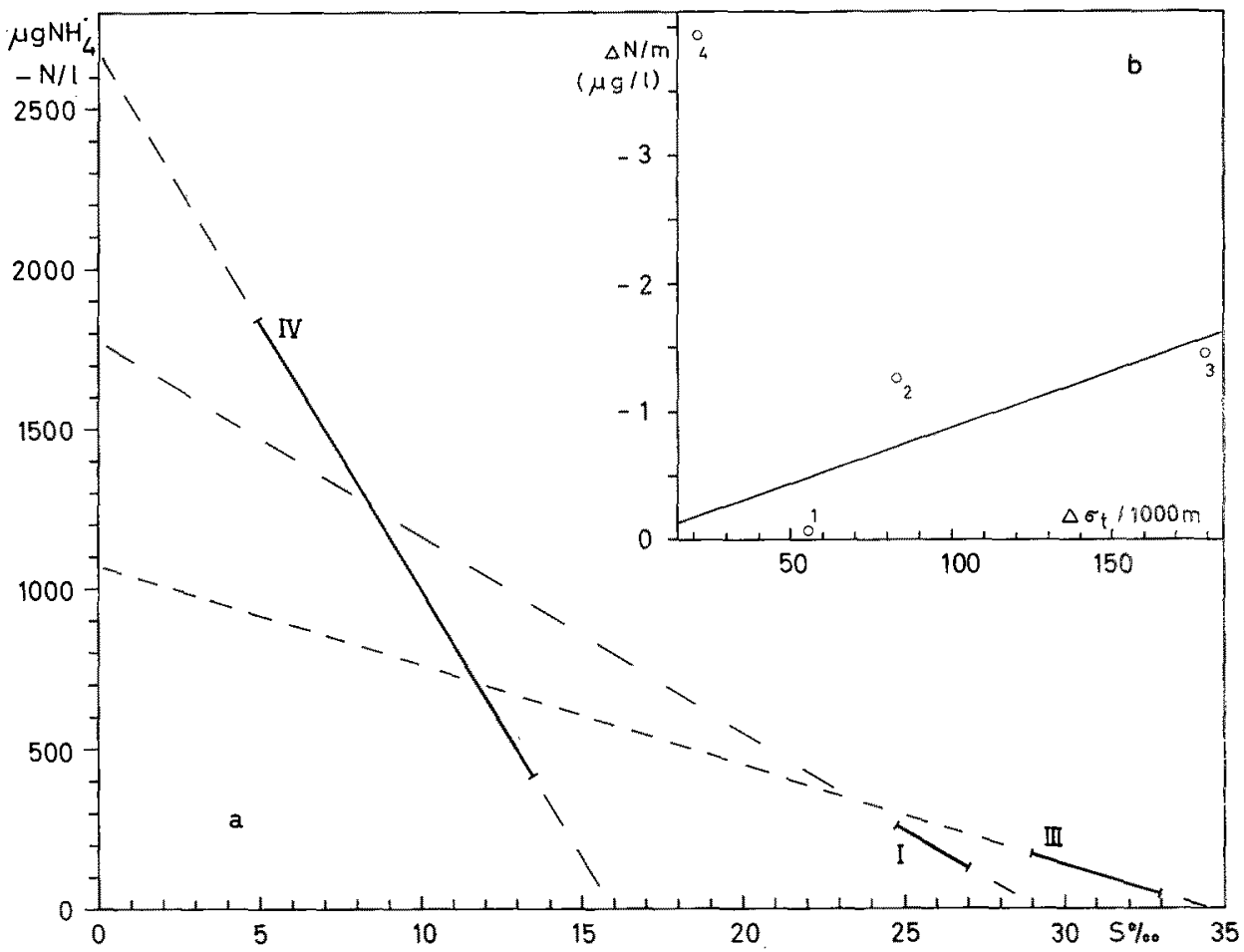

Abb. 4: a) Zusammenhang zwischen Ammoniak- und Salzgehalt

Gerade I : Küstenwasser am 17. 4. 1961 ( $\mathrm{S}<27 \%$ )

Gerade III: Helgolandwasser am 13. 4. 1961

Gerade IV: Wasser bei Cuxhaven am 29. 3. 1957

Im Beobachtungsbereich sind die Geraden ausgezogen

b) Zusammenhang zwischen Abnahme des Ammoniakgehalts mit der Tiefe (nach Eliminierung des Salzgehalts) $[\triangle \mathrm{N} / \mathrm{m}]$ und Stabilität der Schichtung $\left[\triangle \sigma_{t} / 1000 \mathrm{~m}\right]$. Die den Meßpunkten beigefügten Ziffern beziehen sich auf die zugehörigen Zeilen der Tab. 1

Wir erhalten so zwei Grenzfälle:

a) Bei Helgoland war am 13. 4. 1961 ein Wasser, dessen Ammoniakgehalt nur vom Salzgehalt aber nicht von der Tiefe abhing, während

b) beim Wasser der Stationen 4 bis 6 die Tiefenverteilung entscheidend war. Die übrigen Daten zeigen $Z$ wischenwerte. .

Wie sind diese Befunde zu deuten? Das Ammoniak gelangt also zum Teil von der Küste her in die Nordsee, wobei es nicht unbedingt aus dem Süßwasser zu stammen braucht. Es kann auch im flacheren Wasser entstanden sein, in dem zu dieser Zeit schon mit stärkerem Planktonwachstum gerechnet werden muß. Aber auch im Untersuchunggebiet läßt sich die Bildung von 
Ammoniak wahrscheinlich machen. Als Maß hierfür bietet sich die Abnahme des Ammoniaks mit der Tiefe (nach Eliminierung des Salzgehalts) an (Tab. 1). Nun hängt dieser Wert von der Vermischung, der Produktion in der Oberschicht und der Zehrung ab. Wenn wir über den letzten Punkt auch nichts sagen können, so wissen wir doch, daß zu dieser Jahreszeit abnehmende Vermischung die Produktion begünstigt. Beide Größen lassen sich also bis zu einem gewissen Grade durch den Dichtegradienten beschreiben (Tab. 1). Die Beziehung Ammoniak-/Dichtegradient ist in Abb. $4 \mathrm{~b}$ wiedergegeben. Es läßt sich für 1, 2 und 3 (Tab. 1) tatsächlich ein solcher Zusammenhang erkennen. Eine übertrieben enge Korrelation ist natürlich nicht zu erwarten, da die Werte stark streuen, die Berechnung von linearen Gradienten recht formal ist, und z. B. 3 gar keine „Produktion“ besitzt, sondern aus der Deckschicht ständig neues Ammoniak erhält, aber immerhin ist die Tendenz deutlich. 4 unterliegt hingegen hinsichtlich Vermischung und Produktion als Auftriebsgebiet ganz anderen Gesetzen.

Herrn E. Harms danke ich für die zum Teil recht mühevolle praktische Durchführung der Messungen.

\section{Zusammenfassung}

1) Es wird ein Verfahren zum direkten Nachweis von Ammoniak im Seewasser durch alkalische Oxydation mit Hypobromit beschrieben.

2) Der Meßbereich geht von 0 bis $250 \mu \mathrm{g} \mathrm{NH}{ }_{4}{ }_{4} \mathrm{~N} / 1$ bei einer Genauigkeit von $\pm 5 \mu \mathrm{g} \mathrm{NH}{ }_{4}-\mathrm{N} / 1$.

3) Die Bestimmung wird durch Aminosäuren gestört.

4) Ein Anwendungsbeispiel zeigt für die Deutsche Bucht sehr komplizierte Verhältnisse: Offenbar stammt ein Teil des Ammoniaks aus dem Süßwasser bzw. von der Küste, während ein anderer durch Nitratreduktion oder Zersetzung von organischer Substanz entsteht.

\section{Summary}

1) A method is described for the direct determination of ammonia in sea water by oxydation with hypobromit in an alkaline solution.

2) It is applicable to concentrations of 0 to $250 \mu \mathrm{g} \mathrm{NH}{ }_{4}-\mathrm{N} / 1$ with an accuracy of $\pm 5 \mu \mathrm{g} \mathrm{NH}{ }_{4}-\mathrm{N} / 1$.

3) The determination is interfered by amino acids.

4) A given example shows a very complicated situation for the German Bight: One part of the ammonia seems to come from the fresh water respectively from the coast. An other part is formed by reduction of nitrates or by decomposition of organic substances.

\section{Schriftum}

Buljan, M. (1951): Note on a method for determination of ammonia in sea water. J. $\mathrm{m}$. biol. Ass. 30, 2.

Bursche, E., Kühl, H. und Mann, H. (1958): Hydrochemische Faktoren und Phytoplankton während einer Tide in der Elbmündung bei Cuxhaven. Gewässer und Abwässer H. 20. 
Gillbricht, M. (1956): Die Hydrographie des Jadebusens und der Innenjade. Veröff. Inst. Meeresforsch. Bremerhaven 4.

Koroleff, F. (1959): Colorimetric determination of ammonia in sea water. Vortrag gehalten in Kiel.

Nümann, W. (1949): Kolorimetrische Methoden zur quantitativen Bestimmung von Silikat und organisch wie anorganisch gebundenem Phosphor und Stickstoff im Meerwasser unter Benutzung des Pulfrich-Photometers. Dtsche. Hydrogr. Ztschr, $2,4$.

Riley, J. und Sinhaseni, P. (1957): The determination of ammonia and total ionic inorganic nitrogen in sea water. J. mar. biol. Ass. 36.

Strickland, J. und Parsons, T. (1960): A manual of sea water analysis. Fish. Res. Board Canada Bull. 125. 